\section{Chronic pain}

$\mathrm{W}$ ith approximately $25 \%$ of all Canadians experiencing chronic pain, appropriate pain control will become an ever-increasing issue as our population ages. ${ }^{1}$ This level of pain has a tremendous impact on the patient's quality of life and has a significant social and financial impact on both the patient and the general population.

I am writing to commend Gabrielle Richard et al. for their recently published article on monitoring programs for drugs with potential for abuse or misuse. ${ }^{2}$ I was happy to see interest in the monitoring programs in Canada and the need for a national program to:

- Improve opioid prescribing

- Ensure that patients with legitimate pain are treated appropriately

- Identify patients at risk of abuse and aberrant behaviours

- Provide appropriate services to address these issues

As was shown in this article, each of the current monitoring systems does have some significant weaknesses. One of my major concerns is that the primary focus of each of these programs is not on ensuring that the patient's pain is appropriately managed, but instead on monitoring prescribers and pharmacists. This monitoring for inappropriate prescribing and reprimanding health care professionals has the potential to:

- Make clinicians hesitant to use an appropriate treatment option, as they worry about the ramifications

- Encourage clinicians to select less appropriate treatment options, in order to avoid monitoring

- Potentially decrease the quality of care provided to the increasing number of patients in chronic pain

I work with a large number of pain specialists in Canada and I was happy to share your article with each of them. The monitoring issue is becoming of increasing concern to these professionals and your concise summary was helpful and will hopefully stimulate discussion on this topic between patients, health care professionals and regulators.

Although I will not provide a detailed review of the strengths and weaknesses of each of the monitoring systems, I wanted to draw your attention to a recent letter to the editor written by Dr. T. Cicero in the New England Journal of Medicine, about the effect of the abuse-deterrent formulation of OxyContin. Dr. Cicero discusses interviews with 103 patients who were abusing opioids. With the introduction of the tamper-resistant OxyContin, the number of patients using this product to "get high" fell from $47.3 \%$ to $30 \% .{ }^{3}$ Some regulators would view this as an incredible success and a decrease in abuse behaviours in these patients. What was interesting is that the use of heroin in this population nearly doubled. ${ }^{3}$ Based on this research, this may decrease the validity of your conclusion that a reduction in the number of physicians [prescribing OxyContin] could be an indication of decreased abuse and aberrant behaviours. It seems that many of these patients may simply change to other drugs that are not monitored by these programs.

I feel that all health care professionals would like to effectively manage their patients' pain and reduce their risk of opioid abuse. Monitoring programs have a role in helping to educate clinicians, identify patients at risk and, when designed appropriately, can lead to the optimal management of pain with the lowest risk to the patient. I am hoping through continued discussions that this "ideal" monitoring system will be designed and implemented as a national strategy.

- Michael Boivin

Clinical Pharmacist, CME Developer CommPharm Consulting Inc. Barrie, Ontario

\title{
References
}

1. National Opioid Use Guideline Group. Canadian guideline for safe and effective use of opioids for chronic non-cancer pain. 2010. Available: http:// nationalpaincentre.mcmaster.ca/opioid/ (accessed August 12, 2012).

2. Richard G, Ojala V, Ojala A, et al. Monitoring programs for drugs with potential for abuse or misuse in Canada. Can Pharm J 2012;145:168-70.

3. Cicero TJ, Ellis MS, Surratt HL. Effect of abuse-deterrent formulation of OxyContin. N Engl J Med 2012;367:187-9 